\title{
The Observation on Risk of Ventilator Failure Weaning by Ultrasound bedside Monitoring of Inferior Vena Cava and Lung B Line
}

\author{
Zhangshun Shen'1, Qian Zhao', Hongming Pan², Yangjuan Jia', Jianguo Li ${ }^{1}{ }^{*}$ \\ ${ }^{1}$ Department of Emergency Medicine, Hebei General Hospital, Shijiazhuang, China \\ ${ }^{2}$ Graduate School of Hebei Medical University, Shijiazhuang, China \\ Email: *lijg65@163.com
}

How to cite this paper: Shen, Z.S., Zhao, Q., Pan, H.M., Jia, Y.J. and Li, J.G. (2020) The Observation on Risk of Ventilator Failure Weaning by Ultrasound bedside Monitoring of Inferior Vena Cava and Lung B Line. Open Journal of Preventive Medicine, 10, 267-275.

https://doi.org/10.4236/ojpm.2020.1010019

Received: October 10, 2020

Accepted: October 26, 2020

Published: October 29, 2020

Copyright $\odot 2020$ by author(s) and Scientific Research Publishing Inc. This work is licensed under the Creative Commons Attribution International License (CC BY 4.0).

http://creativecommons.org/licenses/by/4.0/

(c) (i) Open Access

\begin{abstract}
Objective: This study was designed to investigate risk of ventilator weaning by ultrasound bedside monitoring of the width of inferior vena cava (IVC) and the number of lung B-lines (B-lines). Methods: This study was conducted on 106 patients from January 2019 to January 2020 who had mechanical ventilation for more than 48 hours in an emergency care unit. They were clinically stable and had the criteria for weaning from the ventilator. Before Spontaneous Breathing Test (SBT) and $30 \mathrm{~min}$ or $120 \mathrm{~min}$ after SBT, the width of IVC and the number of B-lines in patients were monitored via bedside ultrasound. There were 87 cases of successful weaning as a control group and 19 cases of ventilator failure weaning as a study group. Changes of the width of IVC and the number of B-lines were compared in the different stages of SBT. Results: A total of 106 patients were included in this study. There were 87 cases of ventilator successful weaning and 19 cases failure weaning. The weaning success rate was $82.08 \%$. The width of IVC and the number of $\mathrm{B}$-lines in the study group were higher than those in the control group in same stage of SBT, the difference was statistically significant $(\mathrm{P}<0.05)$, and which increased significantly with time. There was no significant difference in the width of IVC and the number of B-lines on the different stage of SBT in the control group $(P>0.05)$, and significant difference in the study group. Conclusion: The width of IVC and the number of B-lines monitored by bedside ultrasound can assess the risk of ventilator weaning, it may be caused by cardiopulmonary interaction.
\end{abstract}

\section{Keywords}

Ventilator Weaning, Cardiopulmonary Interaction, Bedside Ultrasound, IVC, The Lung B Line (B-Lines), SBT 


\section{Introduction}

Mechanical ventilation is a means of respiratory support in the intensive care unit (ICU), most inpatients in ICU need mechanical ventilation. Ventilator weaning failure increases the length of hospital stay and mortality [1] [2]. SBT and Rapid Shallow Breathing Index (RSBI) are widely used to assess the risk of weaning failure because of its simplicity, noninvasive, no cooperation with patient and bedside measurement [3]. However, the phenomenon of shallow and fast breathing often occurs in respiratory failure and weaning failure due to imbalance between respiratory muscle strength and respiratory load, it has caused reinjury to patients, so it is lag for predicting weaning failure. It turned out that RSB has no advantage over other parameters, and may even prolong the weaning time [4] [5]. Another study showed that $15 \%-30 \%$ of the patients who passed the SBT finally weaning failure [6]. To detect the possibility of weaning failure earlier is important. All possible causes of weaning failure should be thought over, especially those potential and reversible causes. However, there are many causes for weaning failure, which is often the result of the comprehensive influence of respiratory function, cardiac function, psychology, spirit, metabolic nutrition and other factors. How to detect the possibility of weaning failure earlier is worth studying. In this study, we measured the width of IVC and the number of B line of lung by ultrasound, and to explore the effect in ventilator weaning. This study was approved by the hospital ethics committee.

\section{Materials and Methods}

\subsection{Study Object}

\subsubsection{General Information}

In this study, 106 patients with weaning were selected as the study objects who were admitted to the emergency ICU of our hospital from January 2019 to January 2020. There were 64 males and 42 females. The average age was $(71.4 \pm 7.9)$ years. The primary diseases included 39 cases of severe pneumonia or acute respiratory distress syndrome (36.79\%), 27 cases of central nervous system diseases (25.47\%), 14 cases of severe sepsis (13.21\%), 12 cases of accidental injury (11.32\%), 9 cases of cardiovascular diseases $(8.49 \%)$, and 5 cases $(4.72 \%)$. The duration of mechanical ventilation (MV) was $25-152$ hours (87.2 \pm 76.9 ) hours before weaning. Main equipment: ventilator produced by Drager company of Germany.

\subsubsection{Inclusion Criteria [6]}

Before SBT, evaluate whether the patients meet the following criterion: 1) The pathogenic factors of mechanical ventilation were removed or corrected; meet the weaning screening criterion: oxygenation index $\geq 150 \mathrm{mmHg}$; 2) Peep $\leq 5-8$ $\mathrm{cm} \mathrm{H}_{2} \mathrm{O}, \mathrm{FiO}_{2} \leq 0.4 ; \mathrm{pH} \geq 7.25$; for patients with COPD, $\mathrm{pH} \geq 7.25, \mathrm{FiO}_{2} \leq 0.35$; $\mathrm{PaO}_{2} \geq 50 \mathrm{mmHg}$; stable hemodynamics, no or only the use of small doses of vasoactive drugs, such as dopamine $\leq 5 \mathrm{ug} / \mathrm{kg} \cdot \mathrm{min}$ and no active myocardial 
ischemia; patients with stable spontaneous breathing, being conscious, powerful cough and expectoration. They also had enough airway protection ability; their nutritional status was improved, and there was no obvious abdominal distension.

\subsubsection{Exclusion Criteria}

Patients who failed in SBT, those with acute coronary syndrome; delirium can not be corrected; electrolyte disorder or acid-base imbalance, severe malnutrition, and cerebrovascular hypertension.

\subsection{Methods}

Patients who meet the weaning parameters were tested by 30 minutes $\mathrm{T}$-tube test. The standard of the SBT success [7]: RSBI < 105; Respiratory rate (RR) $<35$ times/min; heart rate $<140$ times/min or increase $<20 \%$; no obvious dyspnea or auxiliary respiratory muscle were activated in breathing. After success in SBT, patients were given weaning, and those who failed in SBT were treated by mechanical ventilation again, and were not included in the study groups.

Weaning success parameters [8]: weaning time $>48 \mathrm{~h}$; weaning failure parameters [7]: RR > 35 times/min or auxiliary respiratory muscle were activated; $\mathrm{SpO}_{2}<90 \%$; heart rate $>130$ times/min or increase $>20 \%$; mental status changes. According to whether the weaning success or not within 48 hours, the study objects were divided into weaning success group (the control group) and weaning failure group (the study group).

\subsection{Observation Indicators}

Age, Acute Physiology and Chronic Health Evaluation II (APACHE II), body mass index (BMI), oxygenation index, hemoglobin and lactate levels before SBT were recorded. The width of IVC and the number of B-lines were monitored by bedside ultrasound before (SBT), 30 minutes and 120 minutes after the spontaneous breathing test. The average of the width of IVC and the number of B-lines at different time points in each group were calculated, and changes of them were compared in the different stages of SBT.

\subsubsection{The Width of IVC}

The patient took the supine position, the width of IVC was measured by the convex array probe $(2-5 \mathrm{MHz})$ from the lower xiphoid longitudinal section at $0.5-1 \mathrm{~cm}$ distal to the opening of the hepatic vein [9].

\subsubsection{The Number of Lung B Line}

The patient took the supine position, numbers of B-lines were measured by the convex array probe $(2-5 \mathrm{MHz})$ between 2 and 4 intercostals at points of sternal line, median clavicle line, axillary front line, axillary midline. The B-lines is a dynamic artifact, and moving or changing shape with the respiratory cycle. Numbers of which were judged by more than 2 experienced doctors with visual 
inspection [10].

\subsection{Statistical Analysis}

Spss21.0 statistical software was used for statistical analysis. The measurement data were expressed by mean \pm standard deviation $(\bar{X} \pm S)$. T test was used for comparison between the two groups. The difference was statistically significant with $\mathrm{P}<0.05$.

\section{Result}

\subsection{Comparison of the Fundamental State between the Two Groups}

A total of 106 subjects were included in this study. There were 87 cases of ventilator successful weaning as a control group and 19 cases of ventilator failure weaning as a study group. The weaning success rate was $82.08 \%$. There was no significant difference in age, APACHE II score at admission, BMI, oxygenation index, hemoglobin and lactate levels between the two groups $(\mathrm{P}>0.05$, Table 1$)$.

\subsection{The Width of IVC}

The width of IVC before SBT in study group was higher than that in the control group, but no difference was statistically significant $(\mathrm{P}>0.05)$. At $30 \mathrm{~min}$ and 120 min after SBT, the width of IVC in study group was higher than that in the control group, and the difference was statistically significant $(\mathrm{P}<0.05)$. The width of IVC increased gradually in the study group with time, the change was statistically significant $(\mathrm{P}<0.05)$ and no difference was statistically significant in the control group $(\mathrm{P}>0.05)$ (Table 2$)$.

\subsection{The Number of B-Lines}

The Number of B-lines before SBT in study group was higher than that in the control group, but no difference was statistically significant $(P>0.05)$. At $30 \mathrm{~min}$ and 120 min after SBT, the number of B-lines in study group was higher than that in the control group, and the difference was statistically significant $(\mathrm{P}<$ $0.05)$. The number of B-lines increased gradually in the study group with time, the change was statistically significant $(\mathrm{P}<0.05)$ and no difference was statistically significant in the control group (Table 3 ).

\section{Discussion}

In this study, a total of 106 patients were included, and 19 cases of ventilator failure weaning. The weaning success rate was $82.08 \%$. It was similar to previous reports [6]. Some data show that weaning failure is an independent risk factor for prolonged hospital stay and increased mortality in ICU patients [1] [2]. To detect the possibility of weaning failure earlier is important. However, there are many causes for weaning failure, clinicians often have some difficulties in subjective judgment of the causes of weaning failure. Another study such as 
Table 1. Comparison of the fundamental state between the two groups $(\overline{\mathrm{X}} \pm \mathrm{S})$.

\begin{tabular}{cccc}
\hline Item & $\begin{array}{c}\text { Control group } \\
(\mathrm{n}=87)\end{array}$ & $\begin{array}{c}\text { Study group } \\
(\mathrm{n}=19)\end{array}$ & P value \\
\hline age (year) & $72.4 \pm 7.8$ & $72.8 \pm 7.5$ & $\mathrm{P}>0.05$ \\
males/females & 1.52 & 1.54 & $\mathrm{P}>0.05$ \\
BMI (kg/m $\left.{ }^{2}\right)$ & $21.24 \pm 4.78$ & $20.88 \pm 5.05$ & $\mathrm{P}>0.05$ \\
oxygenation index $(\mathrm{mmHg})$ & $185 \pm 32$ & $183 \pm 35$ & $\mathrm{P}>0.05$ \\
hemoglobin $(\mathrm{g} / \mathrm{L})$ & $91 \pm 5.6$ & $89 \pm 6.1$ & $\mathrm{P}>0.05$ \\
APACHE II & $18.7 \pm 3.9$ & $19.1 \pm 4.3$ & $\mathrm{P}>0.05$ \\
lactic acid $(\mathrm{mmol} / \mathrm{L})$ & $1.34 \pm 0.43$ & $1.28 \pm 0.48$ & $\mathrm{P}>0.05$ \\
\hline
\end{tabular}

Table 2. Comparison of the width of IVC between the two groups in different stages $(\overline{\mathrm{X}} \pm \mathrm{S})$.

\begin{tabular}{|c|c|c|c|}
\hline Groups & Before SBT & 30 min after SBT & 120 min after SBT \\
\hline contral group $(n=87)$ & $14.07 \pm 0.44^{\circ}$ & $14.25 \pm 0.61^{\circ}$ & $14.96 \pm 0.70^{\circ}$ \\
\hline study group $(\mathrm{n}=19)$ & $14.62 \pm 0.85^{\mathbf{\Lambda}}$ & $17.93 \pm 1.32^{\star 凶}$ & $19.42 \pm 2.10^{\star 凶}$ \\
\hline
\end{tabular}

${ }^{\star} P<0.05$, compared with contral groups; ${ }^{\mathbf{\Lambda}} P<0.05$, compared with different stages in study group; ${ }^{\circ} P>$ 0.05 , compared with different stages in contral group.

Table 3. Comparison of B-lines of the lungs between the two groups in different stages $(\overline{\mathrm{X}} \pm \mathrm{S})$.

\begin{tabular}{cccc}
\hline Groups & Before SBT & 30 min after SBT & 120 min after SBT \\
\hline contral group $(\mathrm{n}=87)$ & $1.0 \pm 1.7^{\circ}$ & $1.0 \pm 1.8^{\circ}$ & $1.2 \pm 1.4^{\circ}$ \\
study group $(\mathrm{n}=19)$ & $1.1 \pm 1.62^{\star}$ & $2.1 \pm 2.0^{\star}$ & $3.7 \pm 2.6^{\star}$ \\
\hline
\end{tabular}

${ }^{\star} P<0.05$, compared with contral groups; ${ }^{\boldsymbol{\Delta}} P<0.05$, compared with different stages in study group; ${ }^{\circ} P>$ 0.05 , compared with different stages in contral group.

central venous oxygen saturation and brain natriuretic peptide (BNP) levels had been used to assess the risk of weaning failure [11] [12], but no most effective method was found.

Compared with them, bedside ultrasound is a rapidly developing diagnosis technology in critical illness, which is widely used in the fields of hemodynamics and respiratory monitoring [13] [14]. Evaluation of weaning failure has its unique advantages. Ultrasound monitoring width of IVC and B-lines has many advantages such as non-invasive, dynamic, real-time, simple and repeatable operation. At the same time, the dynamic contrast of width of IVC and number of B-lines of the patients themselves can better exclude the influence of other factors on weaning failure. In this study, these two indicators were taken as the breakthrough point, whether there were changes in cardiac function and volume 
state before the occurrence of rapid shallow breathing in weaning, and more strict assessment and adjustment were carried out in advance. Real-time monitoring of the width of IVC and the number of B-lines by bedside ultrasound can effectively guide the volume management, improve the rate of successful weaning, and has strong clinical value.

IVC is a volume vessel directly adjacent to the right atrium, and its inner diameter often changes with the change of body capacity. The width and variability of IVC can effectively evaluate volume status and volume responsiveness [15] [16] [17], which is widely used to guide fluid resuscitation in shock. In this study, at $30 \mathrm{~min}$ and $120 \mathrm{~min}$ after SBT, the width of IVC in study group was higher than that in the control group, and the difference was statistically significant $(\mathrm{P}<0.05)$. The width of IVC increased gradually in the study group with time, the change was statistically significant $(P<0.05)$ and no statistically significant in the control group $(\mathrm{P}>0.05)$ (Table 2).

Lung B-lines is a kind of reverberation artifact on ultrasonic images. It refers to the strong echo when ultrasound meets the strong reflection interface and the narrow strip echo with gradual attenuation and multiple reflection behind it. It is shaped like "comet tail" and a prompt for the level of extravascular lung water [18] [19], and which sensitivity is significantly better than that of chest X-ray film [20]. This study found that the number of B-lines at $30 \mathrm{~min}$ and $120 \mathrm{~min}$ after SBT in study group was higher than that in the control group, and the difference was statistically significant $(\mathrm{P}<0.05)$. The number of B-lines increased gradually in the study group with time, the change was statistically significant ( $\mathrm{P}$ $<0.05$ ) and no statistically significant in the control group (Table 3 ).

This study found that there was a certain difference in the width of IVC and the number of B-lines between the two groups before weaning, and the difference continued to exist after SBT test and after weaning, and the difference increased significantly. It is speculated that the negative pressure of chest cavity increases significantly due to spontaneous breathing after weaning. The heart and lung of the human are in the chest cavity together, and have an anatomic proximity. In the process of respiratory physiology, ventilation and circulation interact by the changes of intrathoracic pressure and lung volume [21], it is called cardiopulmonary interaction, and in which the amount of return heart blood increased, and the left ventricular transmural pressure increased, leading to the cardiac preload and afterload. The cardiopulmonary interaction has already existed in healthy people, and this interaction will be amplified and even cause pathophysiological changes in patients with cardiopulmonary disease. If the patient's heart function reserve is insufficient and cannot be compensated, it will further lead to left heart failure, then produce pulmonary congestion or even pulmonary edema, and finally fail to wean.

In the process of mechanical ventilation, due to the continuous positive pressure of chest, the amount of return heart blood flowing from systemic circulation to right ventricle is reduced, and then the circulation blood volume from 
pulmonary circulation to left ventricle is significantly reduced, left ventricular filling pressure and preload are reduced. At the same time, left ventricular transmural pressure and left ventricular afterload are reduced due to the increase of chest pressure. At the same time, mechanical ventilation can reduce respiratory muscle work and improve cardiac function, which is the theoretical basis of mechanical ventilation in the treatment of left ventricular failure. The most obvious physiological change of patients with mechanical ventilation during spontaneous breathing after weaning is the change of intrathoracic pressure. During inhalation, the volume of blood returned to the heart increased significantly due to the suction of negative pressure in the chest, and the blood flow through the pulmonary circulation to the left ventricle increased. Studies have shown that the cardiac index of patients before and after weaning significantly increased [22]. After weaning, the decrease of chest pressure leads to the increase of left ventricular transmural pressure and the increase of left ventricular posterior negative pressure. The patients have obvious increase in the amount of return heart blood, and even have cardiac dysfunction or insufficient compensation of cardiac function. Clinically, we can see the phenomenon of shallow and fast breathing. The phenomenon of shallow and fast breathing is that the patients can not tolerate the pathophysiological changes after weaning, which leads to heart failure, extravascular lung water increase, and even pulmonary edema, which leads to weaning failure.

\section{Conclusion}

There was a greater possibility of weaning failure in patients with mechanical ventilation who passed the SBT. From the perspective of cardiopulmonary interaction, dynamic monitoring of width of IVC and the number of B-lines may be early predictors for weaning failure risk.

\section{Limitations}

This study is clinical observation with a small sample, so it is not enough for predicting the weaning failure risk in the whole one, which is affected by many factors. Next, we will further expand the sample size and conduct a more in-depth prospective study, in order to provide strong clinical evidence for guiding evaluation of weaning failure risk.

\section{Conflicts of Interest}

The authors declare no conflicts of interest regarding the publication of this paper.

\section{References}

[1] Tonnelier, A., Tonnelier, J.M., Nowak, E., et al. (2011) Clinical Relevance of Classification According to Weaning Difficulty. Respiratory Care, 56, 583-590. https://doi.org/10.4187/respcare.00842 
[2] Jeong, B.H., Ko, M.G., Nam, J., et al. (2015) Differences in Clinical Outcomes According to Weaning Classifications in Medical Intensive Care Units. PLoS ONE, 10, e0122810. https://doi.org/10.1371/journal.pone.0122810

[3] Tobin, M.J. and Jubran, A. (2008) Meta-Analysis under the Spotlight: Focused on Meta-Analysis of Ventilator Weaning. Critical Care Medicine, 36, 1-7. https://doi.org/10.1097/01.CCM.0000297883.04634.11

[4] Boutou, A.K., Abatzidou, F., Tryfon, S., et al. (2011) Diagnostic Accuracy of the Rapid Shallow Breathing Index to Predict a Successful Spontaneous Breathing Trial Outcome in Mechanically Ventilated Patients with Chronic Obstructive Pulmonary Disease. Heart \& Lung, 40, 105-110. https://doi.org/10.1016/j.hrtlng.2010.02.002

[5] Fadaii, A., Amini, S.S., Bagheri, B., et al. (2012) Assessment of Rapid Shallow Breathing Index as a Predictor for Weaning in Respiratory Care Unit. Tanaffos, 11, 28-31. https://pubmed.ncbi.nlm.nih.gov/25191425

[6] Qiu, H.B. and Yu, K.J. (2012) Critical Care Medicine. People's Medical Publishing House, Beijing, 162-165.

[7] Liu, D.W. (2013) Practice of Critical Care Medicine. People's Medical Publishing House, Beijing, 128-129.

[8] Wang, C.-Y., Fan, X.-P., Weng, F.-Z., et al. (2017) Influencing Factors of Ventilator Weaning Failure in COPD Patients Complicated with Respiratory Failure Undergoing Invasive Mechanical Ventilation. Practical Journal of Cardiac Cerebral Pneumal and Vascular Disease, 25, 46-49.

[9] Chinese Critical Ultrasound Study Group (CCUSG), Zhang, Q., Liu, D.W., Wang, X.T., Zhang, H.M., He, H.W., Chai, W.Z. and Chao, Y.G. (2015) A Pilot Study of Measuring Inferior Vena Cava Internal Diameter on Transverse Section Plane in Different Sites by Ultrasonography. Chinese Journal of Internal Medicine, 54, 491-495.

[10] Li, C., Xu, J. and Yu, X.Z. (2013) Lung Ultrosonography for the Evaluation of Extravascular Lung Water. Journal of Clinical Emergency, 14, 559-562.

[11] Wang, W.-J., Xu, L., Feng, Q.-S., et al. (2014) Clinical Research on Predicting Outcome of Difficult-to-Wean by Central Venous Oxygen Saturation. Shandong Medical Journal, 54, 12-16.

[12] Zhou, P., Dong, Y., Chang, L., et al. (2013) Role of Measurement of Serum Brain Natriuretic Peptide in Prediction of Mechanical Ventilation Weaning Failure. Practical Journal of Clinical Medicine, 10, 69-71.

[13] Yang, M., Li, H., Yin, L., et al. (2017) The Value of Bedside Lung Ultrasound Evaluation during the Weaning from Mechanical Ventilation in ICU Patients. Chinese Journal of Critical Care Medicine, 37, 1000-1004.

[14] Assaad, S., Kratzert, W.B., Shelley, B., et al. (2018) Assessment of Pulmonary Edema: Principles and Practice. Journal of Cardiothoracic and Vascular Anesthesia, 32, 901-914. https://doi.org/10.1053/j.jvca.2017.08.028

[15] Monnet, X. and Teboul, J.L. (2013) Assessment of Volume Responsiveness during Mechanical Ventilation: Recent Advances. Critical Care, 17, 217. https://doi.org/10.1186/cc12526

[16] Ferrada, P., Anand, R.J., Whelan, J., et al. (2012) Qualitative Assessment of the Inferior Vena Cava: Useful Tool for the Evaluation of Fluid Status in Critically Ill Patients. The American Surgeon, 78, 468-470.

https://pubmed.ncbi.nlm.nih.gov/22472406

https://doi.org/10.1177/000313481207800439 
[17] Zhang, Z., Xu, X., Ye, S., et al. (2014) Uhrasonographic Measurement of the Respiratory Variation in the Inferior Vena Cava Diameter Is Predictive of Fluid Responsiveness in Critically Ill Patients: Systematic Review and Meta Analysis. Ultrasound in Medicine \& Biology, 40, 845-853.

https://doi.org/10.1016/j.ultrasmedbio.2013.12.010

[18] Scali, M.C., Zagatina, A., Simova, I., et al. (2017) B-Lines with Lung Ultrasound: The Optimal Scan Technique at Rest and During Stress. Ultrasound in Medicine \& Biology, 43, 2558-2566. https://doi.org/10.1016/j.ultrasmedbio.2017.07.007

[19] Asano, M., Watanabe, H., Sato, K., et al. (2018) Validity of Ultrasound Lung Comets for Assessment of the Severity of Interstitial Pneumonia. Journal of Ultrasound in Medicine, 37, 1523-1531. https://doi.org/10.1002/jum.14497

[20] Zhu, B., Xi, X.M., Jiang, Q., et al. (2007) The Diagnostic Value of B-Ultrasonography for Acute Lung Interstitial Diseases in ICU. Chinese Journal of Medicine, 42, 45-47.

[21] Grübler, M.R., Wigger, O., Berger, D., et al. (2017) Basic Concepts of Heart-Lung Interactions during Mechanical Ventilation. Swiss Medical Weekly, 147, w14491. https://doi.org/10.4414/smw.2017.14491

[22] Dres, M., Teboul, J.L. and Monnet, X. (2014) Weaning the Cardiac Patient from Mechanical Ventilation. Current Opinion in Critical Care, 20, 493-498.

https://doi.org/10.1097/MCC.0000000000000131 